\title{
The DUB blade goes snicker-snack: Novel ubiquitin cleavage by a Legionella effector protein
}

\author{
Cell Research (2017) 27:845-846. doi:10.1038/cr.2017.80; published online 2 June 2017
}

\begin{abstract}
Recently, a Legionella pneumophila effector protein was shown to have an unprecedented ATP-independent ubiquitin ligase activity that couples phosphoribosylated ubiquitin (PR-
\end{abstract} Ub) to serine residues of host proteins. A new study published in Cell Research by Qiu et al. reveals that another Legionella effector protein, SidJ, catalyzes deubiquitination of PR-Ub by cleavage of the substratelinked phosphodiester bond.

Posttranslational modification of cellular proteins by ubiquitin influences a broad spectrum of cellular functions such as protein degradation by the $26 \mathrm{~S}$ proteasome, membrane protein trafficking, DNA damage responses, and other signaling events [1]. Covalent attachment of the terminal carboxylate group of ubiquitin to target proteins requires ATP and is catalyzed by the action of three enzymes: E1 (ubiquitin-activating enzyme), E2 (ubiquitin-conjugating enzyme), and E3 (ubiquitin ligase). The most prevalent ubiquitin linkage involves its attachment to the $\varepsilon$-amino group of lysine residues in target proteins, resulting in formation of an amide ("isopeptide") bond. Other types of ubiquitin linkages to substrates have been reported too, such as thioester bonds with cysteine residues or oxyester linkages to serine/threonine residues [2].

Deubiquitinating enzymes (DUBs) comprise a family of enzymes that reverse ubiquitination by hydrolytically cleaving ubiquitin from substrates [3]. While one obvious role for deubiquitination is to maintain a pool of free ubiquitin, it also influences numerous cellular pathways by removing ubiquitin from specific target proteins. DUBs belong to either the thiol protease or metalloprotease mechanistic class. Thiol proteases, which possess a nucleophilic cysteine residue in the active site, represent the larger group of DUBs. Metalloprotease DUBs utilize a nucleophilic water ligated to $\mathrm{Zn}^{2+}$ ion in the active site to initiate catalysis [4]. All members of the metalloprotease DUB family studied thus far possess a conserved JAB1/MPN/ MOV34 (JAMM) motif.

Bacteria lack complete versions of the ubiquitin system sensu stricto. $\mathrm{Nev}$ ertheless, many bacterial pathogens have acquired DUBs and E3 ubiquitin ligases, possibly as a result of horizontal gene transfer, which are secreted as effector proteins to interact with ubiquitin signaling pathways of the host during infection [4]. Intracellular Legionella pneumophila, the causal agent of potentially fatal Legionnaire's disease, secretes close to 300 effector proteins through its type IV secretion system during an infection. Eight of the injected proteins have either F-box or U-box domains that redirect the substrate specificity of the host ubiquitin ligation machinery $[5,6]$. Members of the SidE effector family (SdeA, SdeB, $\mathrm{SdeC}$, and SidE) are large proteins that include DUB domains displaying dual specificity for ubiquitin and the ubiquitin-related Nedd8 protein. This DUB activity reduces accumulation of ubiquitinated species on endoplasmic reticulum (ER)-derived Legionellacontaining vacuoles (LCVs), possibly limiting autophagic removal of LCVs from cells [6].

An exciting recent discovery has been the finding that SdeA ubiquitinates substrates by an unconventional mechanism independent of the canonical ubiquitination machinery and ATP [7]. A mono-ADP-ribosyltransferase (mART) domain conserved in all SidE family members catalyzes ADP-ribosylation of Arg42 of ubiquitin and requires only nicotinamide adenine dinucleotide $(\mathrm{NAD}+)$ as a cofactor [7] (Figure 1). A phosphodiesterase (PDE) domain in SdeA catalyzes cleavage of the pyrophosphate bond in ADP-ribosylated ubiquitin, generating phosphoribosylated ubiquitin (PR-Ub) and AMP, and promoting covalent addition of PR-Ub to a substrate serine residue [8] (Figure 1). In addition to auto-ubiquitination of SdeA, the targets of this non-canonical ubiquitination reaction include ERlocalized Rab GTPases, such as Rab33b, and the ER-sculpting reticulon 4 (Rtn4) protein [7, 9].

Qiu and colleagues [10] now report that another L. pneumophila effector protein, SidJ, which is encoded in the same gene cluster as most SdeA-related factors, functions to deconjugate PR-Ub from SdeA substrates (Figure 1). The phosphodiesterase activity of SidJ is critical for bacterial infection. This activity is notable because, while cleavage of non-isopeptide bonds like oxyester and thioester linkages has been described for some DUBs [3], this is the first report of a DUB that can cleave phosphoribosylated ubiquitin linkages. The ubiquitin-protein phosphodiesterase activity provides insight into how SidJ suppresses SdeAinduced toxicity in yeast and mammalian cells [11]. 


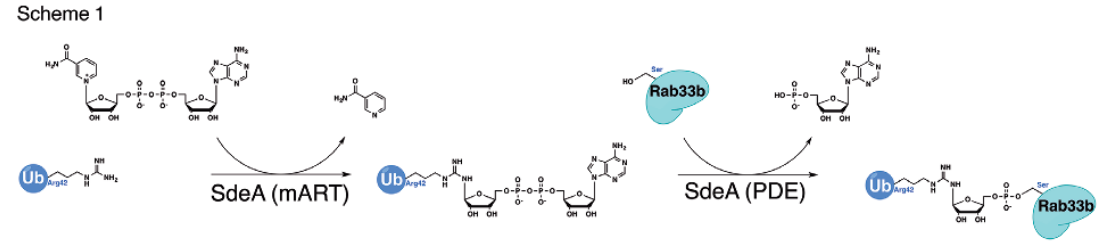

Scheme 2

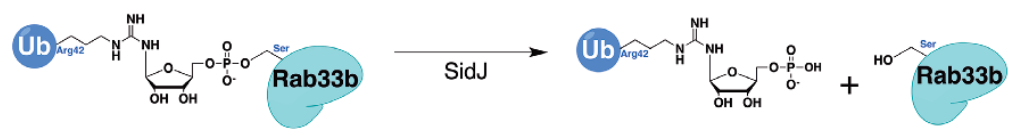

Figure 1 SdeA and SidJ mediate ubiquitination and deubiquitination, respectively, of the Rab33b GTPase. Scheme 1 depicts the unconventional ubiquitin ligase activity of SdeA. First, the mART domain of SdeA utilizes NAD+ to catalyze ADP-ribosylation of Arg42 of ubiquitin, generating an ADP-ribosylated ubiquitin intermediate and releasing nicotinamide. The SdeA PDE domain subsequently catalyzes hydrolysis of the pyrophosphate bond of the ubiquitin intermediate, releasing AMP and enabling transfer of phosphoribosylated ubiquitin to a serine side chain of Rab33b. In Scheme 2, SidJ catalyzes deubiquitination of Rab33b, by a proposed hydrolytic mechanism. Cleavage of the phosphodiester linkage yields unmodified Rab33b and phosphoribosylated ubiquitin. Presumably another enzyme, either from the host or Legionella, can remove the phosphoribosyl group from Arg42 of ubiquitin.

Using a yeast toxicity assay, the authors discovered several SidJ mutants that failed to rescue mART motif-dependent SdeA toxicity and subsequently showed that two of these SidJ mutants accumulated SdeA-ubiquitinated Rab33b (Ub-Rab33b). These data suggested that SidJ suppression of SdeA could be due to a DUB activity. In line with earlier findings [7], the DUB domain of SdeA (residues 1-193) was incapable of cleaving the PR-Ub phosphodiester linkage from Rab33b, suggesting that it does not regulate SdeA ubiquitin ligase activity and that the DUB activity seen here is specific to SidJ. The authors employed mass spectrometric analysis of the ubiquitin species released from Ub-Rab33b treated with SidJ to precisely determine the site of cleavage. The ubiquitin was still phosphoribosylated at Arg42 (Figure 1). Thus, Rab33b is devoid of any PRUb remnants.

By both immunofluorescence and biochemical fractionation assays, the peripheral LCV localization of SdeA is unchanged by its self-ubiquitination or by SidJ deubiquitinase activity. Moreover, through infecting HEK293 cells with various bacterial strains and quantifying levels of Ub-Rab33b over time, the authors found that while the levels of SdeA remain constant, the levels of SidJ increase as the infection progresses. Qiu et al. suggest that high SdeA:SidJ ratios early in infection favor SdeA-mediated ubiquitination of its substrates, whereas during later stages, increasing SidJ levels reverse these modifications, consistent with temporal attenuation of SdeAmediated toxicity by SidJ.

In addition to deubiquitination of PR-Ub-linked substrates, the authors also observed deubiquitination of a mixture of partially purified ubiquitinated proteins presumed to harbor conventional isopeptide ubiquitin linkages. This prompted them to test SidJ activity against a panel of ubiquitin dimers with different linkages (K63, K48, K11, and K33); SidJ exhibits a preference for the K63 linkage. Treatment of SidJ with N-ethylmaleimide, a cysteine-modifying agent, eliminated its DUB activity against both Ub-Rab33b and diubiquitin. However, generation of single point mutations at each of SidJ's three cysteines only partially impaired its
DUB activity, suggesting that SidJ is not a thiol protease. Its amino acid sequence does not bear any obvious JAMM/metalloprotease motifs either, suggesting that SidJ utilizes a novel mechanism for cleavage of isopeptide/phosphoribosyl ubiquitin linkages.

It is not clear whether both DUB activities derive from the same SidJ active site. SidJ incubated with equimolar amounts of K63-linked diubiquitin and (PR)-Ub-Rab33b preferentially cleaves the ubiquitin dimers over the latter. There could be mechanisms that tune one activity relative to the other, possibly by binding of adapter proteins. Structural and biochemical studies will help address these and other questions about this remarkable protein.

Judith A Ronau ${ }^{1}$, Mark Hochstrasser ${ }^{1,2}$

${ }^{1}$ Departments of Molecular Biophysics \& Biochemistry, ${ }^{2}$ Molecular, Cellular, and Developmental Biology, Yale University, 266 Whitney Avenue, New Haven, CT 06520-8114, USA

Correspondence: Mark Hochstrasser

E-mail: mark.hochstrasser@yale.edu

\section{References}

1 Hershko A, Ciechanover A, Varshavsky A. Nat Med 2000; 6:1073-1081.

2 Wang X, Herr RA, Hansen TH. Traffic 2012; 13:19-24.

3 Amerik A, Hochstrasser M. Biochim Biophys Acta 2004; 1695:189-207.

4 Ronau JA, Beckmann JF, Hochstrasser M. Cell Res 2016; 26:441-456.

5 Maculins T, Fiskin E, Bhogaraju S, Dikic I. Cell Res 2016; 26:499-510.

6 Sheedlo MJ, Qiu J, Tan Y, et al. Proc Natl Acad Sci USA 2015; 112:15090-15095.

7 Qiu J, Sheedlo MJ, Yu K, et al. Nature 2016; 533:120-124.

8 Bhogaraju S, Kalayil S, Liu Y, et al. Cell 2016; 167:1636-1649.

9 Kotewicz KM, Ramabhadran V, Sjoblom N, et al. Cell Host Microbe 2017; 21:169-181.

10 Qiu J, Yu K, Fei X, et al. Cell Res 2017; 27:865-881.

11 Jeong KC, Sexton JA, Vogel JP. PLoS Pathog 2015; 11:e1004695. 